\title{
Enhanced Chondrogenesis in Co-Cultures with Articular Chondrocytes and Mesenchymal Stem Cells
}

\author{
Ville V. Meretoja ${ }^{a}$, Rebecca L. Dahlin ${ }^{a}$, F. Kurtis Kaspera ${ }^{a}$, and Antonios G. Mikos ${ }^{a},{ }^{*}$ \\ a Department of Bioengineering, MS-142, Rice University, 6100 Main St., Houston, TX 77005, \\ USA
}

\section{Abstract}

In this work, articular chondrocytes (ACs) and mesenchymal stem cells (MSCs) with 1:1 and 1:3 cell ratios were co-cultured in order to evaluate if a majority of primary ACs can be replaced with MSCs without detrimental effects on in vitro chondrogenesis. We further used a xenogeneic culture model to study if such co-cultures can result in redifferentiation of passaged ACs. Cells were cultured in porous scaffolds for four weeks and their cellularity, cartilage-like matrix formation and chondrogenic gene expression levels (collagen I and II, aggrecan) were measured. Constructs with primary bovine ACs had 1.6 and 5.5 times higher final DNA and glycosaminoglycan contents, respectively, in comparison to those with culture expanded chondrocytes or MSCs harvested from the same animals. Equally robust chondrogenesis was also observed in co-cultures, even when up to $75 \%$ of primary ACs were initially replaced with MSCs. Furthermore, species-specific RT-PCR analysis indicated a gradual loss of MSCs in bovine-rabbit co-cultures. Finally, co-cultures using primary and culture expanded ACs resulted in similar outcomes. We conclude that the most promising cell source for cartilage engineering was the cocultures, as the trophic effect of MSCs may highly increase the chondrogenic potential of ACs thus diminishing the problems with primary chondrocyte harvest and expansion.

\section{Introduction}

Articular cartilage lesions, commonly caused by trauma, erroneous biomechanical conditions or age-related degeneration, have very limited self-regeneration capacity. Due to the low biosynthetic activity in and avascular nature of mature cartilage tissue, even small cartilage lesions fail to heal on their own [1,2]. In cases of deep defects down to the level of subchondral bone, the formation of a blood clot and the recruitment of stem cells can contribute to the healing process. Clinically, this phenomenon is commonly exploited in subchondral drilling and microfracture techniques, where bleeding is artificially induced to help in the treatment of cartilage defects [3, 4]. Furthermore, autologous chondrocyte transplantation, often in combination with a culture substrate, has recently been used to provide enough cartilaginous matrix producing cells to fill relatively large defects. Unfortunately, none of the current treatment methods are able to reliably regenerate native hyaline cartilage structure but usually result in more fibrous and mechanically inferior repair tissue.

\footnotetext{
(C) 2012 Elsevier Ltd. All rights reserved.

*Corresponding author. Tel.: +1 713348 5355; fax: +1 713348 4244. mikos@ rice.edu (A.G. Mikos)..
}

Publisher's Disclaimer: This is a PDF file of an unedited manuscript that has been accepted for publication. As a service to our customers we are providing this early version of the manuscript. The manuscript will undergo copyediting, typesetting, and review of the resulting proof before it is published in its final citable form. Please note that during the production process errors may be discovered which could affect the content, and all legal disclaimers that apply to the journal pertain. 
The interdependent problems of donor site morbidity, lack of availability and rapid dedifferentiation in culture are major drawbacks associated with autologous chondrocyte transplantation. Depending on the size and quality of the original cartilage biopsy, approximately twenty to fifty fold culture expansion of the harvested cells is required to obtain clinically relevant numbers of cells [5]. Alternative cell sources and tissue engineering methods are therefore actively being researched $[6,7]$. One promising cell source for cartilage engineering is mesenchymal stem cells (MSCs). MSCs can be harvested and expanded from various tissue sources including bone marrow, synovium and adipose tissue, with certain phenotypic differences depending on the source and culture conditions $[8,9]$. The most widely utilized tissue source for MSCs is bone marrow, due to the ease of harvest and high chondrogenic potential of marrow-derived MSCs. A major difficulty with MSCs is that they produce cartilage only after extensive chondrogenic induction, which however, can further lead to hypertrophy and calcification, reminiscent of endochondral ossification $[10,11]$.

Lately, co-cultures of articular chondrocytes (ACs) and MSCs have been proposed to mitigate the above mentioned problems. Interactions between the two cell types can maintain their chondrogenic phenotype, resulting in robust chondrogenesis while still minimizing the need for mature chondrocytes. The functionality of AC-MSC co-cultures was originally demonstrated in pellet cultures [12] and only few studies have further evaluated such co-cultures using either hydrogels [13-15] or porous scaffolds [16] as threedimensional tissue engineering matrices. Furthermore, although both primary and culture expanded chondrocytes have been used in the co-cultures, there seem to be no previous studies comparing the two in a single culture model.

The aim of the current study was to evaluate if a majority of primary ACs can be replaced with MSCs without detrimental effects on in vitro chondrogenesis when cultured in porous scaffolds. We further examined whether such co-cultures can rescue the chondrogenic potential of culture expanded ACs.

\section{Materials and Methods}

\subsection{Scaffold preparation}

Nonwoven poly ( $\varepsilon$-caprolactone) (PCL) microfiber mats were fabricated using a horizontal electrospinning setup with a copper ring to stabilize the electric field as previously described [17]. Mats were electrospun to an average fiber diameter of approximately $10 \mu \mathrm{m}(9.6 \pm 2.0$ $\mu \mathrm{m}$ ) using a solution of $18 \mathrm{wt} \%$ PCL (Inherent viscosity range 1.0 - 1.3; Durect corporation, Cupertino, CA) in a 5:1 volume ratio of chloroform to methanol. The polymer solution was pumped at a flow rate of $40 \mathrm{~mL} / \mathrm{h}$ through a blunt $16 \mathrm{G}$ needle, while charged with an applied voltage of $30 \mathrm{kV}$ to draw microfibers toward the collector plate $33 \mathrm{~cm}$ from the needle tip. The resulting PCL mats of approximately $1.3 \mathrm{~mm}$ thickness were aerated, inspected for consistent microfiber morphology using SEM, and stored in a desiccator.

PCL scaffolds were prepared by die-punching 8-mm-diameter discs from the electrospun mats using a dermal biopsy punch. The scaffolds were then sterilized by exposure to ethylene oxide (AN74i, Andersen Sterilizers, Haw River, NC) at ambient temperature for 14 $\mathrm{h}$ and aerated overnight to remove residual fumes. In order to eliminate air bubbles and to improve cell adhesion, scaffolds were prewetted by centrifugaton through a graded series of ethanol $(100 \%-20 \%)$, followed by two rinses in phosphate buffered saline (PBS), and incubation in general culture medium (high-glucose DMEM, $10 \%$ fetal bovine serum (FBS), penicillin/streptomycin/fungizone (PSF)) for three days. 


\subsection{Cell harvest and expansion}

Bovine MSCs and ACs were harvested from 7-10 day old calves (Research 87, Boylston, MA), less than $36 \mathrm{~h}$ after slaughter using previously established protocols [18]. Briefly, bone marrow was harvested from tibiae and femora, diluted with culture medium and separated from fat by centrifugation. Marrow isolations were plated onto culture flasks and allowed to adhere for $48 \mathrm{~h}$ before washing with PBS to remove non-adherent cells and blood clots. Cultures were maintained in general medium until confluent and passaged for sub-cultures. After two passages, MSCs from a minimum of three animals were pooled, aliquoted, and frozen in freezing medium (DMEM with $20 \%$ FBS and $10 \%$ dimethyl sulfoxide). Articular cartilage (from the same animals) was collected from the femoral condyle area, minced to approximately $1 \times 1 \times 1 \mathrm{~mm}$, washed with PBS and digested in chondrocyte culture medium (DMEM, 10\% FBS, $1 \%$ non-essential amino acids, $50 \mu \mathrm{g} / \mathrm{mL}$ ascorbic acid, $46 \mu \mathrm{g} / \mathrm{mL} \mathrm{L-}$ proline, $20 \mathrm{mM}$ HEPES, PSF) containing $2 \mathrm{mg} / \mathrm{mL}$ collagenase type II (Worthington biochemical corporation, Lakewood, NJ) while incubating at $37{ }^{\circ} \mathrm{C}$ overnight. Primary ACs were pooled, aliquoted, and frozen in freezing medium.

Rabbit MSCs were harvested and isolated as previously described [19]. Briefly, bone marrow was aspirated from the tibiae of six 6 month old male New Zealand white rabbits (Myrtle's Rabbitry Inc., Thompsons Sta, TN). Under general anesthesia, bone marrow from each leg was aspirated into a $10 \mathrm{~mL}$ syringe containing $5000 \mathrm{U} / \mathrm{mL}$ heparin to prevent coagulation. The bone marrow was plated in tissue culture flasks and non-adherent cells were washed away after $72 \mathrm{~h}$. Cultures were maintained in general medium until confluent, cells from all animals were pooled together, aliquoted, and frozen in freezing medium.

\subsection{Cell seeding and chondrogenic culture}

Two independent cell cultures were performed: one with primary $\left(\mathrm{p}^{0}\right)$ bovine ACs and $\left(\mathrm{p}^{3}\right)$ bovine MSCs, one with culture expanded $\left(\mathrm{p}^{2}\right)$ bovine ACs and $\left(\mathrm{p}^{2}\right)$ rabbit MSCs. The purpose of the xenogeneic model was to facilitate the cell type specific gene expression analysis of co-cultured chondrocytes and MSCs. In both cases, scaffolds were seeded with pure AC and MSC populations as well as their combinations, with 1:1 and/or 1:3 AC-toMSC ratios.

The frozen AC and MSC aliquots were thawed and sub-cultured as indicated to establish seeding suspensions of $1.25 \times 10^{6}$ cells / $\mathrm{mL}$. In preparation for cell seeding, prewetted scaffolds were press-fitted into cylindrical poly(methyl methacrylate) cassettes designed to confine the seeding suspension. Cells (in $\sim 200 \mu \mathrm{L}$ of general medium) were then pipetted onto each scaffold to achieve a final seeding density of $4 \times 10^{6}$ cells / $\mathrm{mL}$ scaffold volume. Cells were allowed to adhere for $4 \mathrm{~h}$ before culture medium was gently added to completely cover the cassettes. Scaffolds were removed from the cassettes after $24 \mathrm{~h}$ and placed in 12 well culture plates with $4 \mathrm{~mL}$ of serum free chondrogenic medium (high-glucose DMEM, 1 $\%$ ITS+ premix [BD Biosciences, San Jose, CA], $50 \mu \mathrm{g} / \mathrm{mL}$ ascorbic acid, $100 \mathrm{nM}$ dexamethasone, $10 \mathrm{ng} / \mathrm{mL}$ TGF-beta 3 [PeproTech, Rocky Hill, NJ], PSF). Half of the medium was replenished two to three times a week. Four replicate scaffolds were harvested after 0,14 , and 28 days of chondrogenic culture and washed with PBS. A $3 \mathrm{~mm}$ biopsy punch was then used to obtain individual samples for each analysis.

\subsection{Biochemical assays}

Two $3 \mathrm{~mm}$ biopsy samples were pooled together from each scaffold and frozen in $-20{ }^{\circ} \mathrm{C}$ until used for biochemical assays. Thawed samples were digested in $500 \mu \mathrm{L}$ of a proteinase $\mathrm{K}$ solution $(1 \mathrm{mg} / \mathrm{mL}$ proteinase $\mathrm{K}, 0.01 \mathrm{mg} / \mathrm{mL}$ pepstatin A and $0.185 \mathrm{mg} / \mathrm{mL}$ iodoacetamide in a $50 \mathrm{mM}$ tris(hydroxymethyl aminomethane) $-1 \mathrm{mM}$ ethylenediaminetetraacetic acid buffer, $\mathrm{pH} 7.6$ ) in a $56^{\circ} \mathrm{C}$ water bath for $16 \mathrm{~h}$. DNA and 
matrix components were extracted via two additional freeze-thaw cycles followed by $10 \mathrm{~min}$ sonication.

DNA content of the scaffolds was determined using Quant-iT PicoGreen dsDNA Assay Kit (Invitrogen, Eugene, OR). Briefly, cell lysate, assay buffer and dye solution were combined in an opaque 96-well plate in duplicates, and allowed to incubate for $10 \mathrm{~min}$ at room temperature. Fluorescence was measured using excitation and emission wavelengths of 485 $\mathrm{nm}$ and $528 \mathrm{~nm}$ (FL x800 Fluorescence Microplate Reader; BioTek Instruments, Winooski, VT), respectively, and DNA concentrations were determined relative to a lambda DNA standard curve.

Glycosaminoglycan (GAG) content was determined using the colorimetric dimethylmethylene blue assay [20]. Briefly, cell lysate and color reagent were combined in a transparent 96-well plate in duplicates, and allowed to incubate for $7 \mathrm{~min}$ at room temperature. Absorbance at $520 \mathrm{~nm}$ was measured (PowerWave x340 Microplate Reader; BioTek Instruments), and GAG concentrations were determined relative to a chondroitin sulfate standard curve. For glycosaminoglycan synthetic activity, the resulting GAG amounts were normalized to the amount of DNA for each sample.

\subsection{Histology}

One $3 \mathrm{~mm}$ biopsy sample from each scaffold was fixed for histology in $10 \%$ neutral buffered formalin (Fisher Scientific, Pittsburgh, PA), then immersed in 70\% ethanol prior to embedding in HistoPrep freezing medium (Fisher Scientific). Frozen sections $5 \mu \mathrm{m}$ thick were cut using a cryostat (Leica CM 1850 UV; Leica Biosystems Nussloch GmbH, Germany), mounted onto glass slides, and placed on a $42{ }^{\circ} \mathrm{C}$ slide warmer to facilitate adhesion. Sections were stained with Safranin $\mathrm{O}$ to visualize the distribution of cartilaginous extracellular matrix. Images were obtained using a light microscope with a digital camera attachment (Axio Imager.Z2 equipped with AxioCam MRc5; Carl Zeiss MicroImaging GmbH, Germany).

\subsection{Real-time reverse transcription polymerase chain reaction}

After $3 \mathrm{~mm}$ samples of the scaffolds were removed for biochemical analysis and histology, the remainder of the scaffolds were minced to approximately $1 \times 1 \times 1 \mathrm{~mm}$ pieces and used for RNA isolation using an RNeasy mini kit (Qiagen, Valencia, CA). Briefly, minced scaffolds were placed into $600 \mu \mathrm{L}$ of lysis buffer and incubated at room temperature for $30 \mathrm{~min}$ with periodical vortexing. Cell lysate was then passed through a QIAshredder homogenization column and stored at $-80^{\circ} \mathrm{C}$ until further processing. An equal volume of $70 \%$ ethanol was added to thawed lysates and RNA isolation was continued following the animal cell protocol provided by the manufacturer. Reverse transcription was then carried out to synthesize cDNA from purified RNA samples using Oligo(dT) primers (Promega, San Luis Obispo, CA) and SuperScript III reverse transcriptase (Invitrogen, Carlsbad, CA). Finally, cDNA was subjected to real-time PCR (Applied Biosystems 7300 Real-Time PCR System, Foster City, CA) using SYBR Green detection (PerfeCTa SYBR Green FastMix, ROX; Quanta Biosciences, Gaithersburg, MD) with custom designed primers (Integrated DNA Technologies, Coralville, IA).

Primer sequences are given in Table 1. Species specificity (>99\%) and PCR efficiency $(>95 \%)$ of each primer pair was verified before use. Target gene expression was first normalized to the expression of the housekeeping gene GAPDH in the same sample $(\Delta \mathrm{Ct})$, then to the average baseline expression of that target gene measured in the bovine AC group at the start of chondrogenic cultures $(\Delta \Delta \mathrm{Ct})$. Finally, the $2^{-\Delta \Delta \mathrm{Ct}}$ method was used to convert normalized gene expression levels to fold differences and statistics were calculated on these 
values [21]. Similarly, $2^{-\Delta \mathrm{Ct}}$ was used to calculate the ratios of collagen II / collagen I and bovine-specific / cross-species expression within individual samples.

\subsection{Statistics}

Results are presented as means \pm standard deviations. Statistical analysis was performed with an SPSS 16.0 software package (SPSS, Chicago, IL). Biochemical assay data were analyzed using one-way ANOVA followed by Tukey's post-hoc test, whereas RT-PCR data were analyzed using the Kruskal-Wallis test followed by the Mann-Whitney U test. Differences were considered significant at $95 \%$ confidence level.

\section{Results}

\subsection{Scaffold cellularity}

A continuous increase in scaffold cellularity was observed in all culture groups with primary bovine ACs and bovine MSCs, as seen in Figure 1A. The presence of primary chondrocytes enhanced cell proliferation as the DNA content in the beginning of the cultures was the highest but at later times the lowest with pure MSCs. Similarly, increasing cellularity was observed also with passaged bovine chondrocytes whereas DNA content in the pure rabbit MSC group decreased in culture (Figure 1B). Furthermore, there were no differences between primary AC and co-culture groups at any time, whereas the passaged AC group had significantly lower DNA contents in comparison to the corresponding co-culture group.

\subsection{GAG synthesis}

All cultures with primary bovine ACs and bovine MSCs showed continuously increasing GAG contents and synthetic activity, as seen in Figures 2A and 2B. In the beginning of the chondrogenic cultures, GAG synthesis was strongest with the primary AC group, whereas at later times co-cultures reached similar or even higher levels of GAG content and synthesis than pure ACs. With passaged bovine ACs and their co-cultures, GAG contents and synthesis increased during the first two weeks, but then remained at similar levels for the remainder of the cultures (Figure 2C and 2D). The increase was larger with co-cultures than with pure ACs. Rabbit MSCs showed relatively high synthetic activity in the early cultures, whereas GAG contents actually decreased during culture (due to low cellularity).

\subsection{Histology}

Histological analysis showed that cells and extracellular matrix (ECM) accumulated mostly on the upper part of the scaffolds, with increasing cellular infiltration during the culture. Histology corroborated the results of GAG measurements. Primary ACs and their cocultures had similar histological appearances, whereas clearly thinner cell/ECM crust was observed in pure bovine MSC cultures (Figure 3A). In contrast to primary cells, co-cultures with passaged ACs produced much higher tissue contents than ACs alone (Figure 3B). Furthermore, only very limited amounts of cells/ECM were observed with pure rabbit MSCs.

\subsection{RT-PCR}

RT-PCR analysis was performed only with passaged ACs and rabbit MSCs. As expected, the initial gene expression levels for aggrecan and collagen types I and II were lowest with undifferentiated MSCs and highest with pure chondrocytes, and the same was true also for the collagen type II-to-type I expression ratio (Figure 4). MSC and co-culture groups showed strong chondrogenic induction already after two weeks of culture, and after four weeks they had similar or even higher gene expression levels than pure chondrocytes. 
Chondrogenic markers were always higher in co-culture than in the MSC group, except that the highest aggrecan expression after four weeks was seen in MSCs.

Species-specific analysis of gene expression showed a strong continuous decrease in the proportion of rabbit MSCs in the co-cultures. In the beginning, the majority of GAPDH and collagen I signal came from rabbit MSCs, whereas collagen II and aggrecan expression were predominantly of bovine origin (Figure 5). This was consistent with the relative expression levels in pure AC and MSC populations combined with the 1:3 AC-to-MSC seeding ratio. The situation had changed already after two weeks and all studied markers indicated a mostly bovine cell population, with further increase in bovine specific signal at the end of the culture period.

\section{Discussion}

Cell-based cartilage engineering strategies involve the harvesting and in vitro expansion of chondrogenic cells for subsequent implantation usually within a porous scaffold or embedded in a carrier matrix to repair the original cartilage defect. The most commonly used cell types are articular chondrocytes and bone marrow derived mesenchymal stem cells. Challenges with chondrocytes include lack of availability and rapid dedifferentiation upon culturing, whereas MSCs require extensive induction by exogenously added growth factors to obtain the chondrogenic phenotype, which can also lead to hypertrophy and calcification. The current study aimed to investigate different cell sources for chondrogenic cultures, and to explore the potential of AC-MSC co-cultures for cartilage engineering.

In this study, serum-free differentiation medium supplemented with chondrogenic factors was used to create a controlled culture environment suitable for multiple cell types. This kind of defined medium is commonly applied for MSC differentiation, and may decrease experimental variation related to unknown serum composition [22]. While serum-free conditions can limit chondrocyte proliferation, the medium still allows for efficient cartilaginous ECM production [23, 24]. Furthermore, although the electrospun PCL scaffolds used in the current study may not be optimal for mechanically demanding applications, they provide an excellent 3D culture model for cartilage engineering.

Much higher DNA and matrix contents were observed with primary chondrocytes than with passage two cells, consistent with the notion of rapid dedifferentiation of culture expanded chondrocytes [25-27]. In addition, passaged ACs showed decreasing collagen expression and unchanged GAG contents from two to four weeks, further highlighting the problem of dedifferentiation during prolonged cultures. Primary chondrocytes have been previously shown to produce superior engineered cartilage in comparison to MSCs harvested from the same animals $[18,28]$, and this was also the case in the current study. In contrast, the outcome with passaged ACs seemed to be similar to that with MSCs. Interestingly, two recent studies with large animal models demonstrated equal or even better cartilage repair using MSCs than culture expanded chondrocytes [29, 30]. On the other hand, it has been shown that chondrocytes can retain their original phenotype or even redifferentiate in cocultures with primary cells, potentially increasing their applicability in cartilage engineering [31-33].

Bovine and rabbit MSCs showed distinctly different behavior in the current culture model. Bovine MSCs showed clear proliferation and matrix formation, whereas rabbit cultures indicated decreasing cellularities and GAG contents, similar to our previous study [19]. Although the viability of rabbit MSCs decreased in culture, the cells obtained a highly chondrogenic phenotype. Most importantly, both MSC types induced chondrogenesis in the co-cultures to levels similar to or even higher than with pure chondrocytes. Gene expression 
analysis indicated progressive loss of rabbit MSCs also in co-cultures, suggesting that MSCs functioned primarily as inducers of AC proliferation and redifferentiation. This was consistent with other studies where matrix related genes were predominantly expressed by chondrocytes even when MSCs were retained in the co-cultures [12, 14, 34]. In addition, it was recently proposed that predifferentiating MSCs toward the osteogenic lineage might further enhance chondrogenesis in AC-MSC co-cultures [15]. Cultured MSCs produce a multitude of cytokines and growth factors [35], including known chondrogenic inducers like TGF-betas [36], BMP-2 [37] and IGF-1 [38]. It seems that this trophic effect is the main driving force in AC-MSC co-cultures, although direct cell-cell contacts also contribute to the improved chondrogenesis [34]. Furthermore, the trophic effect is not limited to the culture model used in the current study, but can be observed with several different cell sources, species and culture conditions [39]. In contrast, other studies have shown that chondrocytes can induce chondrogenic differentiation of co-cultured MSCs and that those MSCs might also directly contribute to cartilage formation [16, 40, 41]. It should be noted that the few clinical trials done with MSCs have all used undifferentiated cells within a carrier matrix, therefore relying mostly on the host response to the implanted constructs [42, 43].

We tested two different AC-to-MSC cell ratios in the bovine co-cultures, namely 1:1 and $1: 3$. This range was selected based on current literature $[34,44]$ and proved to result in comparable outcomes, without any significant difference between the two. This suggests that the majority of primary ACs, the golden standard for cartilage engineering, can be replaced by MSCs without affecting the cartilage-like matrix formation. Clinically relevant numbers of cells with chondrogenic potential are more easily obtained from bone marrow than from cartilage biopsy, and decreasing the demand for primary ACs by lowering the proportion of chondrocytes in the co-culture should therefore be beneficial. In addition, MSCs are known to be immunosuppressive and could potentially provide an allogeneic cell source in the future $[45,46]$. Importantly, although primary cells were superior in monocultures, the co-culture results were similar with primary and culture expanded ACs, demonstrating further potential to lessen the requirements for AC harvest. One additional benefit of AC-MSC co-cultures, which however was not evaluated in the current study, is the capacity to decrease hypertrophy and mineralization often seen in chondrogenically induced MSC constructs [13, 41, 47]. This effect, however, may be lost in constructs with a high proportion of MSCs [14, 48], and the AC-to-MSC cell ratio should therefore be kept within the optimal range.

\section{Conclusions}

In this work, we investigated chondrogenesis in porous scaffolds using primary and culture expanded ACs, MSCs and combinations thereof as potential cell sources for cartilage tissue engineering. Engineered constructs with primary ACs had higher cellularities and cartilagelike matrix formation in comparison to those with culture expanded chondrocytes or MSCs harvested from the same animals. Equally robust chondrogenesis was also observed in cocultures, even when up to $75 \%$ of ACs were replaced with MSCs. Most importantly, cocultures using primary and culture expanded ACs resulted in similarly positive outcomes. The most promising cell source for cartilage engineering was the co-cultures, as the trophic effect of MSCs may highly increase the chondrogenic potential of ACs thus diminishing the problems with primary chondrocyte harvest and expansion.

\section{Acknowledgments}

This study was supported by the National Institutes of Health grant R01 AR057083. 


\section{References}

1. Chu CR. Chondral and osteochondral injuries: Mechanisms of injury and repair responses. Oper Tech Orthop. 2001; 11:70-75.

2. Mankin HJ. The response of articular cartilage to mechanical injury. J Bone Joint Surg Am. 1982; 64:460-466. [PubMed: 6174527]

3. Falah M, Nierenberg G, Soudry M, Hayden M, Volpin G. Treatment of articular cartilage lesions of the knee. Int Orthop. 2010; 34:621-630. [PubMed: 20162416]

4. Farr J, Cole B, Dhawan A, Kercher J, Sherman S. Clinical cartilage restoration evolution and overview. Clin Orthop. 2011; 469:2696-2705. [PubMed: 21240578]

5. Brittberg M, Peterson L, Sjogren-Jansson E, Tallheden T, Lindahl A. Articular cartilage engineering with autologous chondrocyte transplantation - a review of recent developments. J Bone Joint Surg Am. 2003; 85A:109-115. [PubMed: 12925617]

6. Chung C, Burdick JA. Engineering cartilage tissue. Adv Drug Deliv Rev. 2008; 60:243-262. [PubMed: 17976858]

7. Temenoff JS, Mikos AG. Review: Tissue engineering for regeneration of articular cartilage. Biomaterials. 2000; 21:431-440. [PubMed: 10674807]

8. Hildner F, Albrecht C, Gabriel C, Redl H, van Griensven M. State of the art and future perspectives of articular cartilage regeneration: A focus on adipose-derived stem cells and platelet-derived products. J Tissue Eng Regen Med. 2011; 5:E36-E51. [PubMed: 21413156]

9. O'Sullivan J, D'Arcy S, Barry FP, Murphy JM, Coleman CM. Mesenchymal chondroprogenitor cell origin and therapeutic potential. Stem Cell Res Ther. 2011:2. [PubMed: 21251334]

10. Dickhut A, Pelttari K, Janicki P, Wagner W, Eckstein V, Egermann M, et al. Calcification or dedifferentiation: Requirement to lock mesenchymal stem cells in a desired differentiation stage. J Cell Physiol. 2009; 219:219-226. [PubMed: 19107842]

11. Farrell E, Both SK, Odorfer KI, Koevoet W, Kops N, O'Brien FJ, et al. In-vivo generation of bone via endochondral ossification by in-vitro chondrogenic priming of adult human and rat mesenchymal stem cells. BMC Musculoskelet Disord. 2011:12. [PubMed: 21232150]

12. Tsuchiya K, Chen GP, Ushida T, Matsuno T, Tateishi T. The effect of coculture of chondrocytes with mesenchymal stem cells on their cartilaginous phenotype in vitro. Mater Sci Eng C. 2004; 24:391-396.

13. Bian L, Zhai DY, Mauck RL, Burdick JA. Coculture of human mesenchymal stem cells and articular chondrocytes reduces hypertrophy and enhances functional properties of engineered cartilage. Tissue Eng Part A. 2011; 17:1137-1145. [PubMed: 21142648]

14. Mo XT, Guo SC, Xie HQ, Deng L, Zhi W, Xiang Z, et al. Variations in the ratios of co-cultured mesenchymal stem cells and chondrocytes regulate the expression of cartilaginous and osseous phenotype in alginate constructs. Bone. 2009; 45:42-51. [PubMed: 18708174]

15. Rothenberg AR, Ouyang L, Elisseeff JH. Mesenchymal stem cell stimulation of tissue growth depends on differentiation state. Stem Cells Dev. 2011; 20:405-414. [PubMed: 20887213]

16. Liu X, Sun HY, Yan D, Zhang L, Lv XJ, Liu TY, et al. In vivo ectopic chondrogenesis of bmscs directed by mature chondrocytes. Biomaterials. 2010; 31:9406-9414. [PubMed: 21056466]

17. Pham QP, Sharma U, Mikos AG. Electrospun poly(epsilon-caprolactone) microfiber and multilayer nanofiber/microfiber scaffolds: Characterization of scaffolds and measurement of cellular infiltration. Biomacromolecules. 2006; 7:2796-2805. [PubMed: 17025355]

18. Mauck RL, Yuan X, Tuan RS. Chondrogenic differentiation and functional maturation of bovine mesenchymal stem cells in long-term agarose culture. Osteoarthritis Cartilage. 2006; 14:179-189. [PubMed: 16257243]

19. Liao JH, Guo XA, Grande-Allen KJ, Kasper FK, Mikos AG. Bioactive polymer/extracellular matrix scaffolds fabricated with a flow perfusion bioreactor for cartilage tissue engineering. Biomaterials. 2010; 31:8911-8920. [PubMed: 20797784]

20. Farndale RW, Buttle DJ, Barrett AJ. Improved quantitation and discrimination of sulfated glycosaminoglycans by use of dimethylmethylene blue. Biochim Biophys Acta. 1986; 883:173177. [PubMed: 3091074] 
21. Livak KJ, Schmittgen TD. Analysis of relative gene expression data using real-time quantitative pcr and the $2^{\wedge}$ (-delta delta ct) method. Methods. 2001; 25:402-408. [PubMed: 11846609]

22. Heng BC, Cao T, Lee EH. Directing stem cell differentiation into the chondrogenic lineage in vitro. Stem Cells. 2004; 22:1152-1167. [PubMed: 15579636]

23. Byers BA, Mauck RL, Chiang IE, Tuan RS. Transient exposure to transforming growth factor beta 3 under serum-free conditions enhances the biomechanical and biochemical maturation of tissueengineered cartilage. Tissue Eng Part A. 2008; 14:1821-1834. [PubMed: 18611145]

24. Freyria AM, Cortial D, Ronziere MC, Guerret S, Herbage D. Influence of medium composition, static and stirred conditions on the proliferation of and matrix protein expression of bovine articular chondrocytes cultured in a 3-d collagen scaffold. Biomaterials. 2004; 25:687-697. [PubMed: 14607507]

25. Darling EM, Athanasiou KA. Rapid phenotypic changes in passaged articular chondrocyte subpopulations. J Orthop Res. 2005; 23:425-432. [PubMed: 15734258]

26. Kang SW, Yoo SP, Kim BS. Effect of chondrocyte passage number on histological aspects of tissue-engineered cartilage. Biomed Mater Eng. 2007; 17:269-276. [PubMed: 17851169]

27. Salzmann GM, Sauerschnig M, Berninger MT, Kaltenhauser T, Schonfelder M, Vogt S, et al. The dependence of autologous chondrocyte transplantation on varying cellular passage, yield and culture duration. Biomaterials. 2011; 32:5810-5818. [PubMed: 21592563]

28. Huang AH, Stein A, Tuan RS, Mauck RL. Transient exposure to transforming growth factor beta 3 improves the mechanical properties of mesenchymal stem cell-laden cartilage constructs in a density-dependent manner. Tissue Eng Part A. 2009; 15:3461-3472. [PubMed: 19432533]

29. Li WJ, Chiang H, Kuo TF, Lee HS, Jiang CC, Tuan RS. Evaluation of articular cartilage repair using biodegradable nanofibrous scaffolds in a swine model: A pilot study. J Tissue Eng Regen Med. 2009; 3:1-10. [PubMed: 19004029]

30. Marquass B, Schulz R, Hepp P, Zscharnack M, Aigner T, Schmidt S, et al. Matrix-associated implantation of predifferentiated mesenchymal stem cells versus articular chondrocytes in vivo results of cartilage repair after 1 year. Am J Sports Med. 2011; 39:1401-1412. [PubMed: 21527412]

31. Ahmed N, Gan L, Nagy A, Zheng JN, Wang C, Kandel RA. Cartilage tissue formation using redifferentiated passaged chondrocytes in vitro. Tissue Eng Part A. 2009; 15:665-673. [PubMed: 18783319]

32. Tan AR, Dong EY, Andry JP, Bulinski JC, Ateshian GA, Hung CT. Coculture of engineered cartilage with primary chondrocytes induces expedited growth. Clin Orthop. 2011; 469:27352743. [PubMed: 21267800]

33. Taylor DW, Ahmed N, Gan L, Gross AE, Kandel RA. Proteoglycan and collagen accumulation by passaged chondrocytes can be enhanced through side-by-side culture with primary chondrocytes. Tissue Eng Part A. 2010; 16:643-651. [PubMed: 19754222]

34. Wu L, Leijten JCH, Georgi N, Post JN, van Blitterswijk CA, Karperien M. Trophic effects of mesenchymal stem cells increase chondrocyte proliferation and matrix formation. Tissue Eng Part A. 2011; 17:1425-1436. [PubMed: 21247341]

35. Sekiya I, Vuoristo JT, Larson BL, Prockop DJ. In vitro cartilage formation by human adult stem cells from bone marrow stroma defines the sequence of cellular and molecular events during chondrogenesis. Proc Nat Acad Sci USA. 2002; 99:4397-4402. [PubMed: 11917104]

36. Grimaud E, Heymann D, Redini F. Recent advances in tgf-beta effects on chondrocyte metabolism - potential therapeutic roles of tgf-beta in cartilage disorders. Cytokine Growth Factor Rev. 2002; 13:241-257. [PubMed: 12486877]

37. Sailor LZ, Hewick RM, Morris EA. Recombinant human bone morphogenetic protein-2 maintains the articular chondrocyte phenotype in long-term culture. J Orthop Res. 1996; 14:937-945. [PubMed: 8982137]

38. Elder BD, Athanasiou KA. Systematic assessment of growth factor treatment on biochemical and biomechanical properties of engineered articular cartilage constructs. Osteoarthritis Cartilage. 2009; 17:114-123. [PubMed: 18571441] 
39. Wu L, Prins H-J, Helder MN, van Blitterswijk CA, Karperien M. Trophic effects of mesenchymal stem cells in chondrocyte co-cultures are independent of culture conditions and cell sources. Tissue Eng Part A. 2011 Ahead of print (doi: 10.1089/ten.tea.2011.0715).

40. Acharya C, Adesida A, Zajac P, Mumme M, Riesle J, Martin I, et al. Enhanced chondrocyte proliferation and mesenchymal stromal cells chondrogenesis in coculture pellets mediate improved cartilage formation. J Cell Physiol. 2012; 227:88-97. [PubMed: 22025108]

41. Aung A, Gupta G, Majid G, Varghese S. Osteoarthritic chondrocyte-secreted morphogens induce chondrogenic differentiation of human mesenchymal stem cells. Arthritis Rheum. 2011; 63:148158. [PubMed: 20954186]

42. Koga H, Engebretsen L, Brinchmann JE, Muneta T, Sekiya I. Mesenchymal stem cell-based therapy for cartilage repair: A review. Knee Surg Sports Traumatol Arthrosc. 2009; 17:1289_ 1297. [PubMed: 19333576]

43. ClinicalTrials.gov. A phase i/ii study of chondrogen delivered by intra-articular injection following meniscectomy. Online. 2012. Available from URL: http://clinicaltrials.gov/ct2/show/NCT00225095?term=chondrogen\&rank=2

44. Zhang L, Li Q, Zhou G, Liu Y, Liu W, Zhang W, et al. Co-culture of microtic chondrocyte with bmsc to generate tissue engineered cartilage. Tissue Eng Part A. 2011 Ahead of print (doi: 10.1089/ten.TEA.2011.0443).

45. Caplan A. Why are mscs therapeutic? New data: New insight. J Pathol. 2009; 217:318-324. [PubMed: 19023885]

46. Le Blanc K, Ringden O. Immunomodulation by mesenchymal stem cells and clinical experience. J Intern Med. 2007; 262:509-525. [PubMed: 17949362]

47. Fischer J, Dickhut A, Rickert M, Richter W. Human articular chondrocytes secrete parathyroid hormone-related protein and inhibit hypertrophy of mesenchymal stem cells in coculture during chondrogenesis. Arthritis Rheum. 2010; 62:2696-2706. [PubMed: 20496422]

48. Pound JC, Green DW, Chaudhuri JB, Mann S, Roach HI, Oreffo ROC. Strategies to promote chondrogenesis and osteogenesis from human bone marrow cells and articular chondrocytes encapsulated in polysaccharide templates. Tissue Eng. 2006; 12:2789-2799. [PubMed: 17518648] 

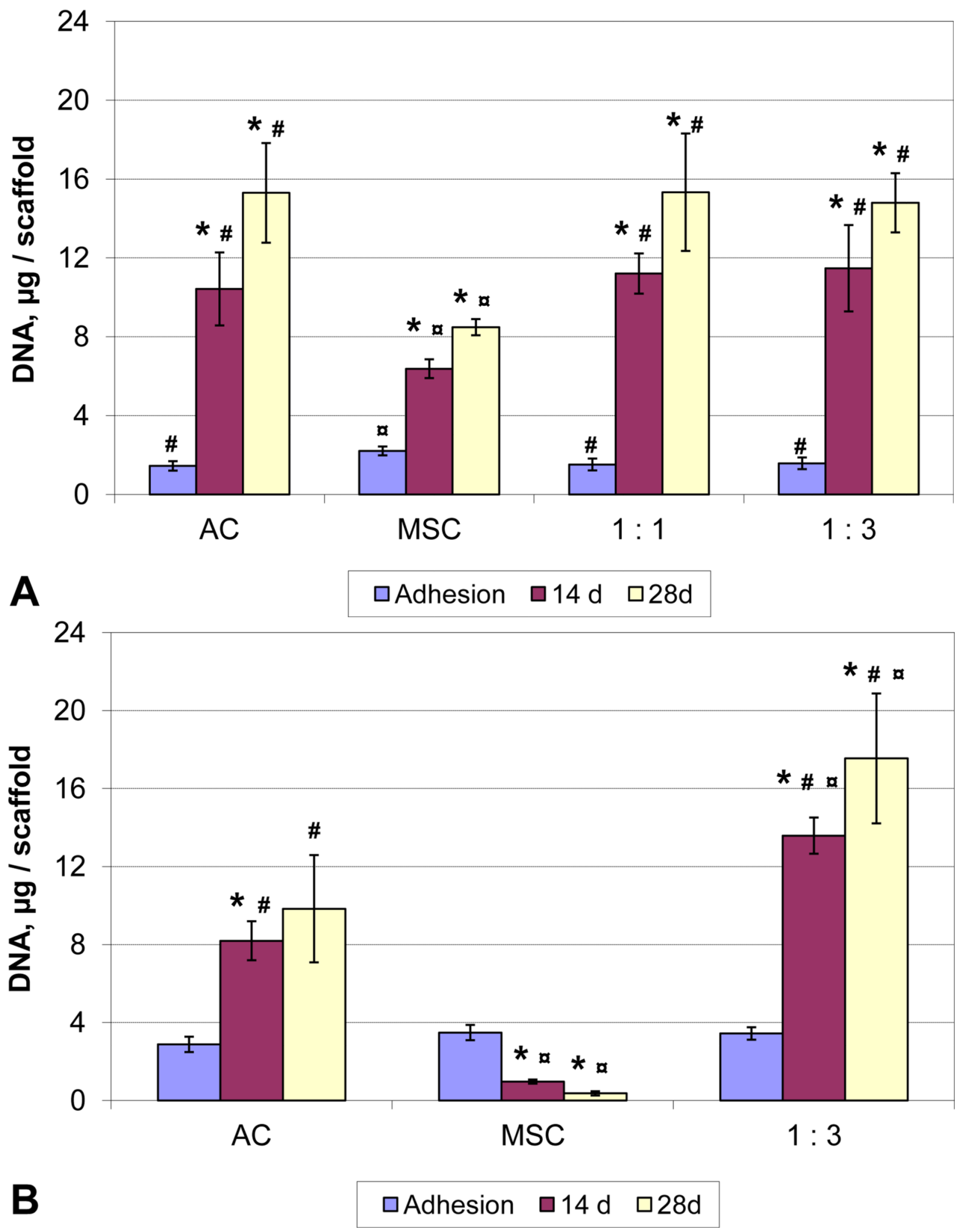

Figure 1. 
DNA content of cultured constructs with primary bovine ACs and bovine MSCs (A) and with expanded bovine ACs and rabbit MSCs (B). Results are presented as mean \pm SD with $n$ $=4$. $\#, \not$ and $*$ denote statistically significant difference to the corresponding MSC and AC culture and to the previous time point, respectively $(p<0.05)$. 

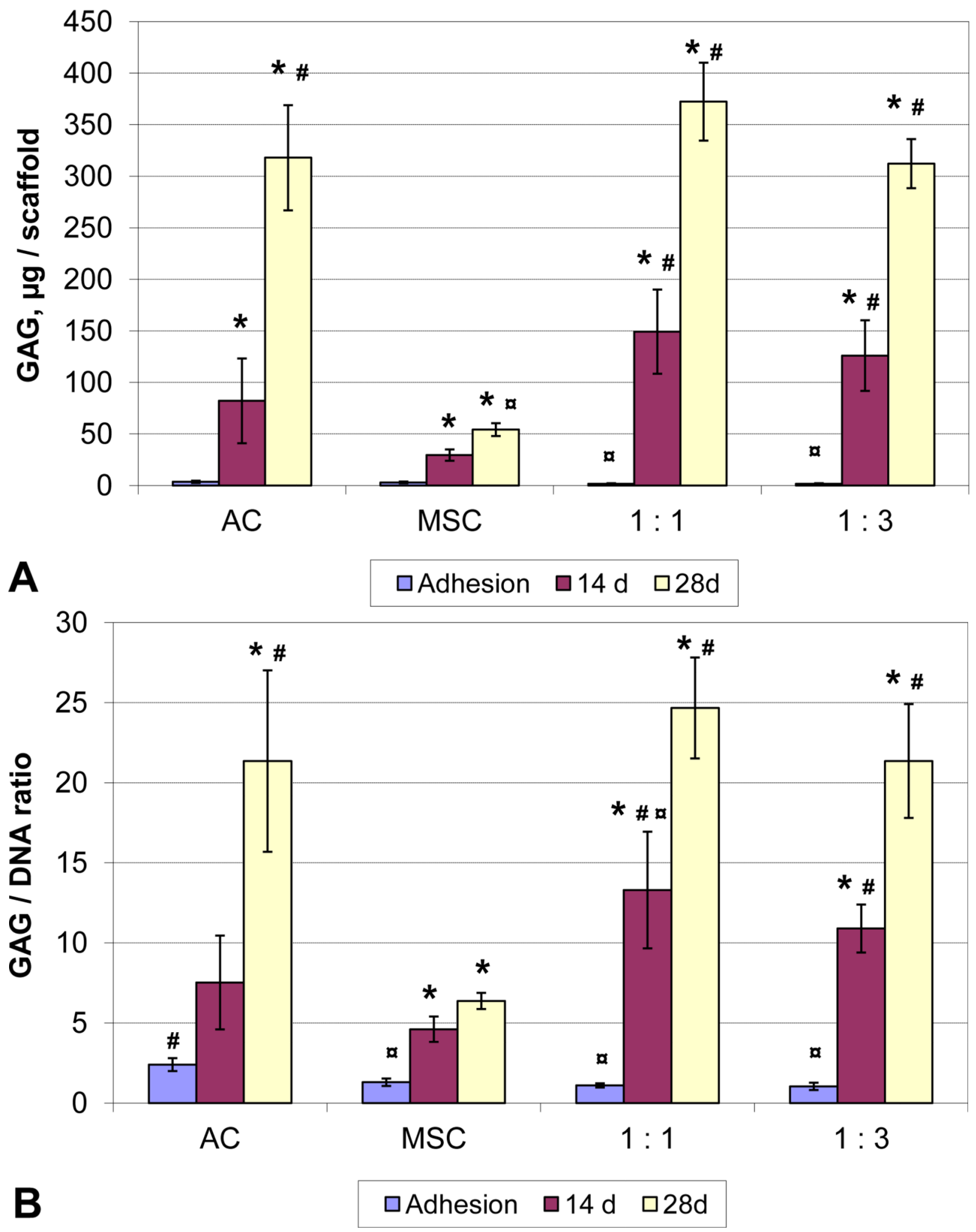

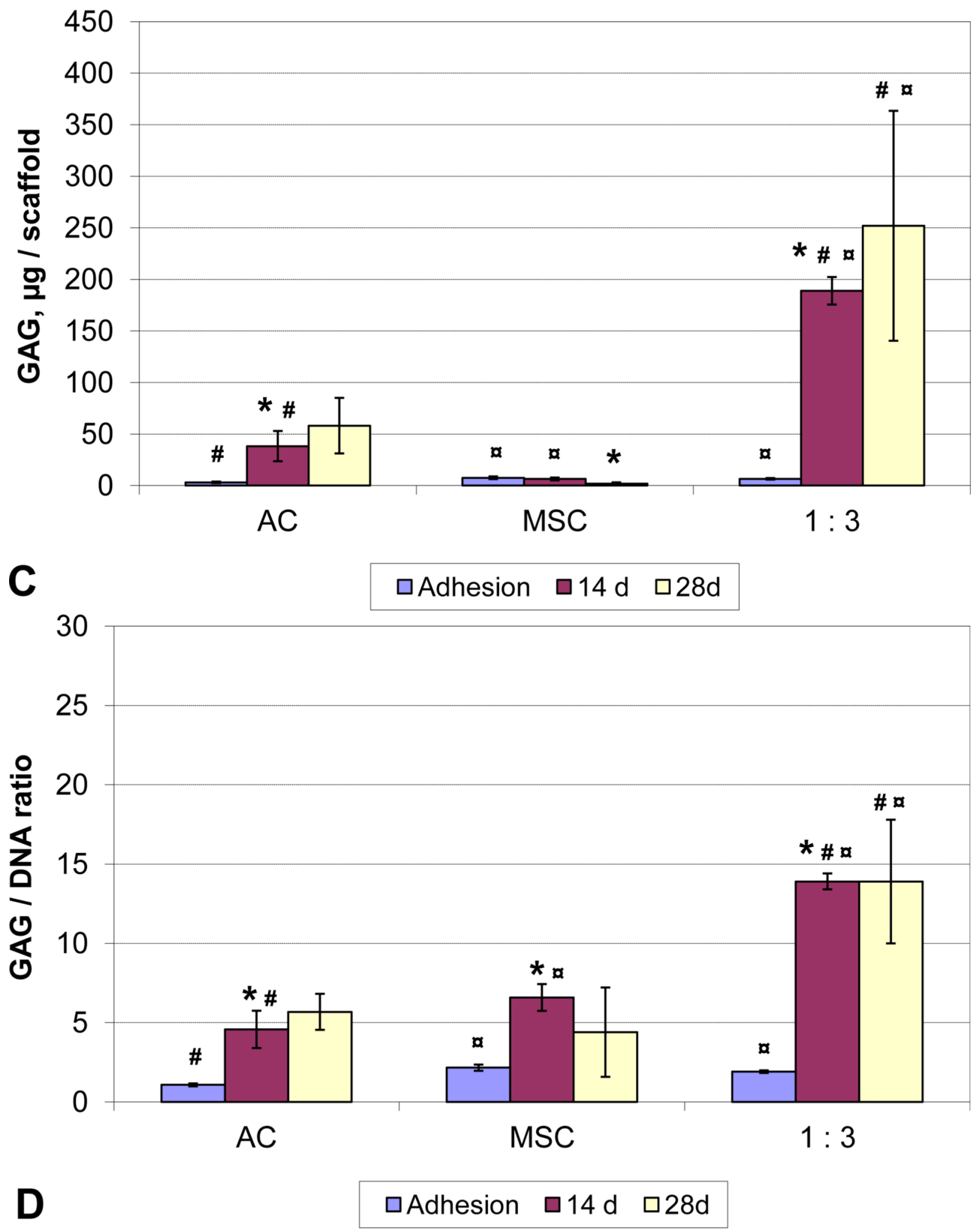

Figure 2.

GAG content (A, C) and synthetic activity (B, D) of cultured constructs with primary bovine ACs and bovine MSCs (A, B) and with expanded bovine ACs and rabbit MSCs (C, D). 
Results are presented as mean \pm SD with $n=4$. \#, a and $*$ denote statistically significant difference to the corresponding MSC and AC culture and to the previous time point, respectively $(p<0.05)$. 


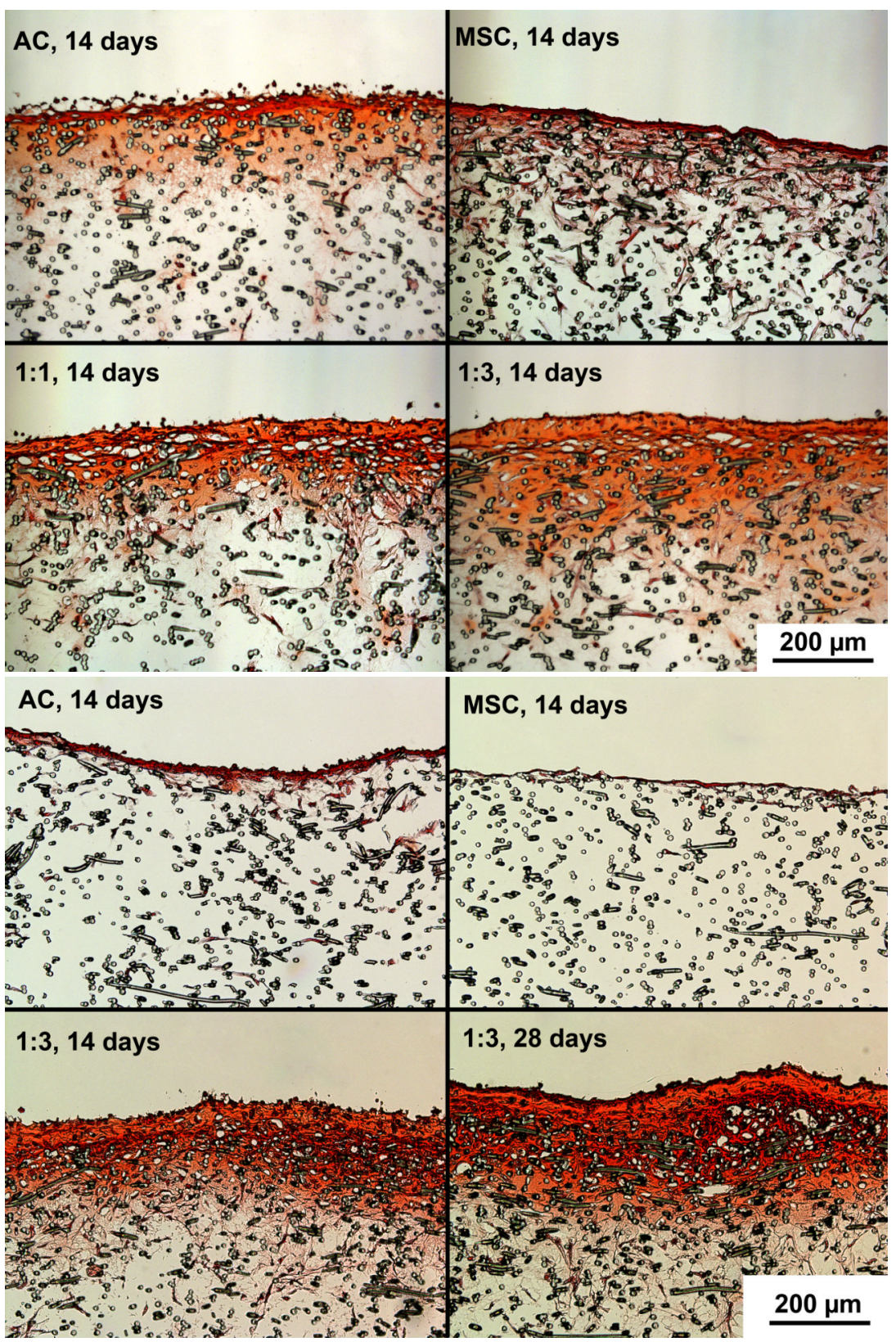

Figure 3.

Cartilage-like matrix production within cultured constructs with primary bovine ACs and bovine MSCs (left panel) and with expanded bovine ACs and rabbit MSCs (right panel). Images taken after 14 days of culture unless otherwise stated. Scale bar represents $200 \mu \mathrm{m}$. Safranin-O staining. 


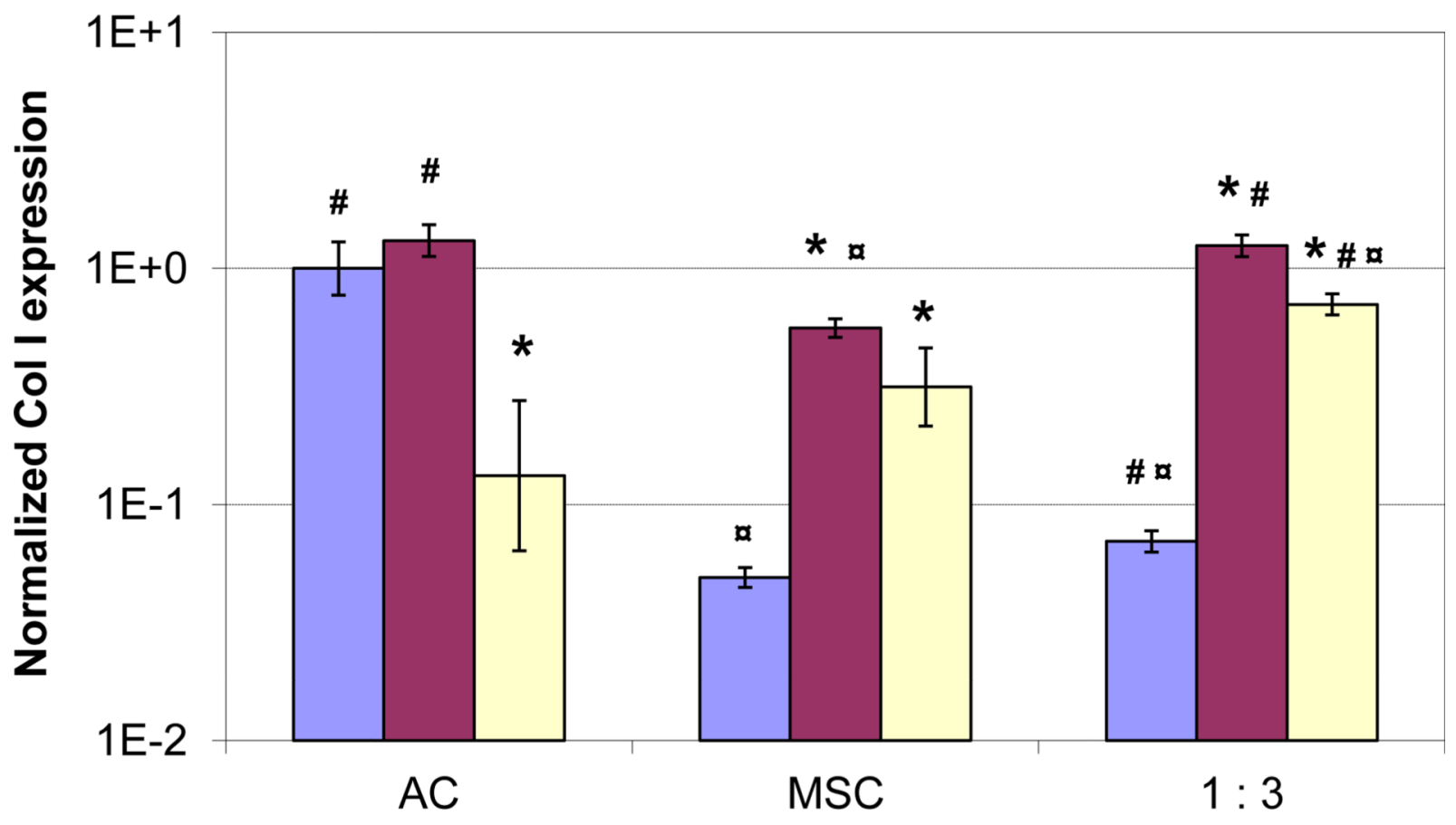

$\square$ Adhesion $\square 14$ days $\square 28$ days

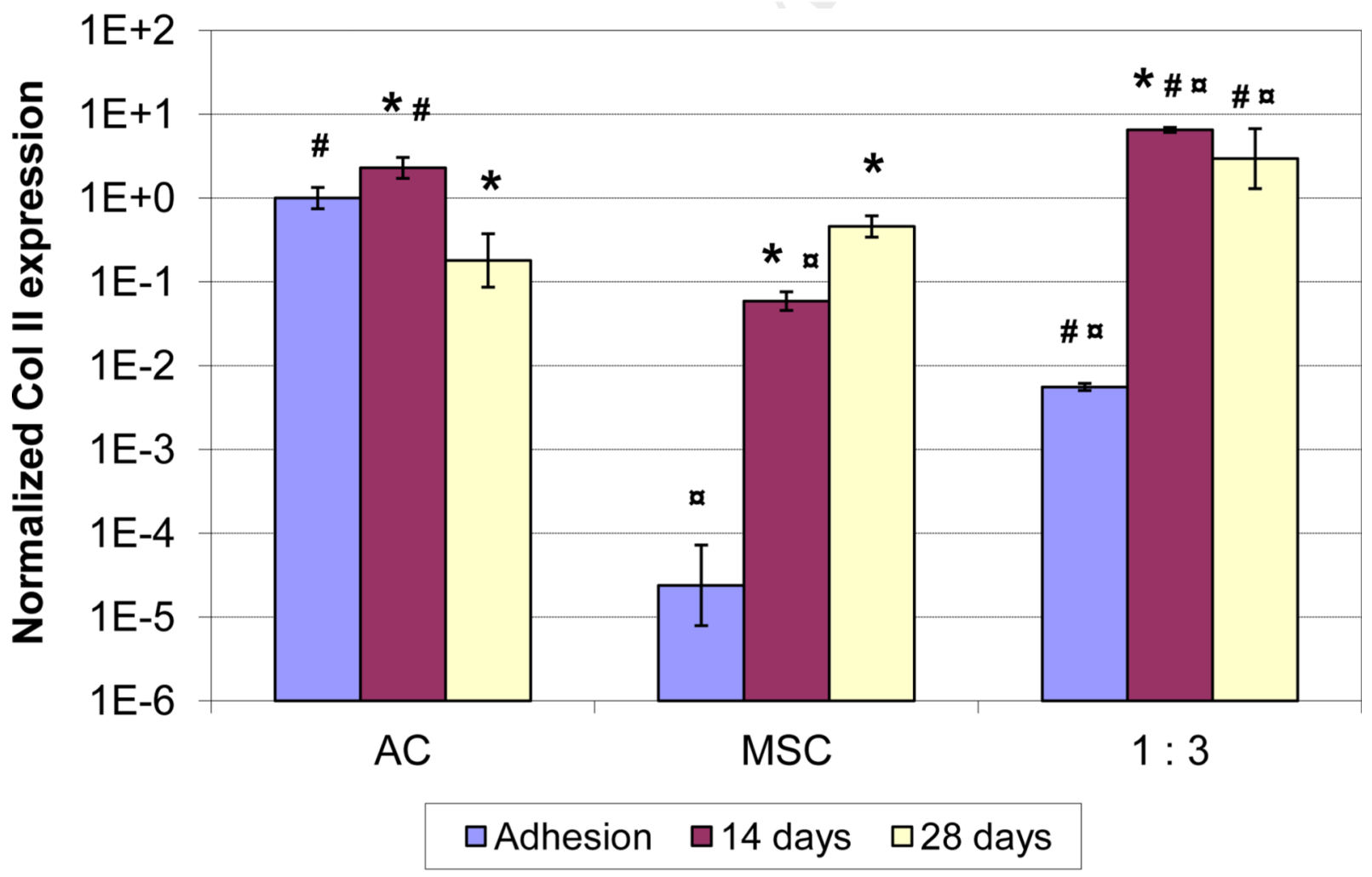




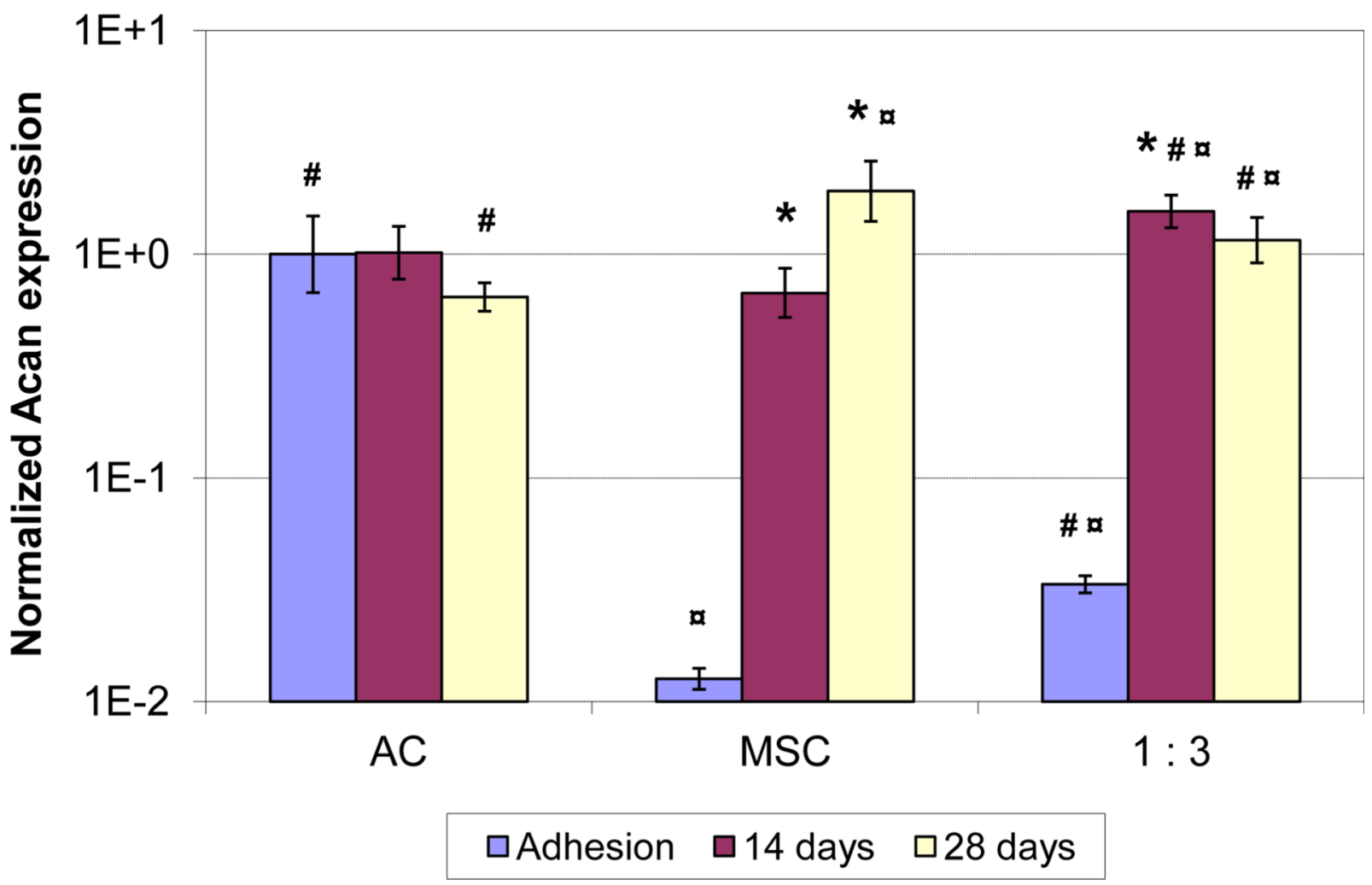

Figure 4.

Quantitative gene expression results for expanded bovine ACs and rabbit MSCs. Results are presented as mean \pm SD with $\mathrm{n}=4$. \#, a and $*$ denote statistically significant difference to the corresponding MSC and AC culture and to the previous time point, respectively ( $p<$ $0.05)$. 

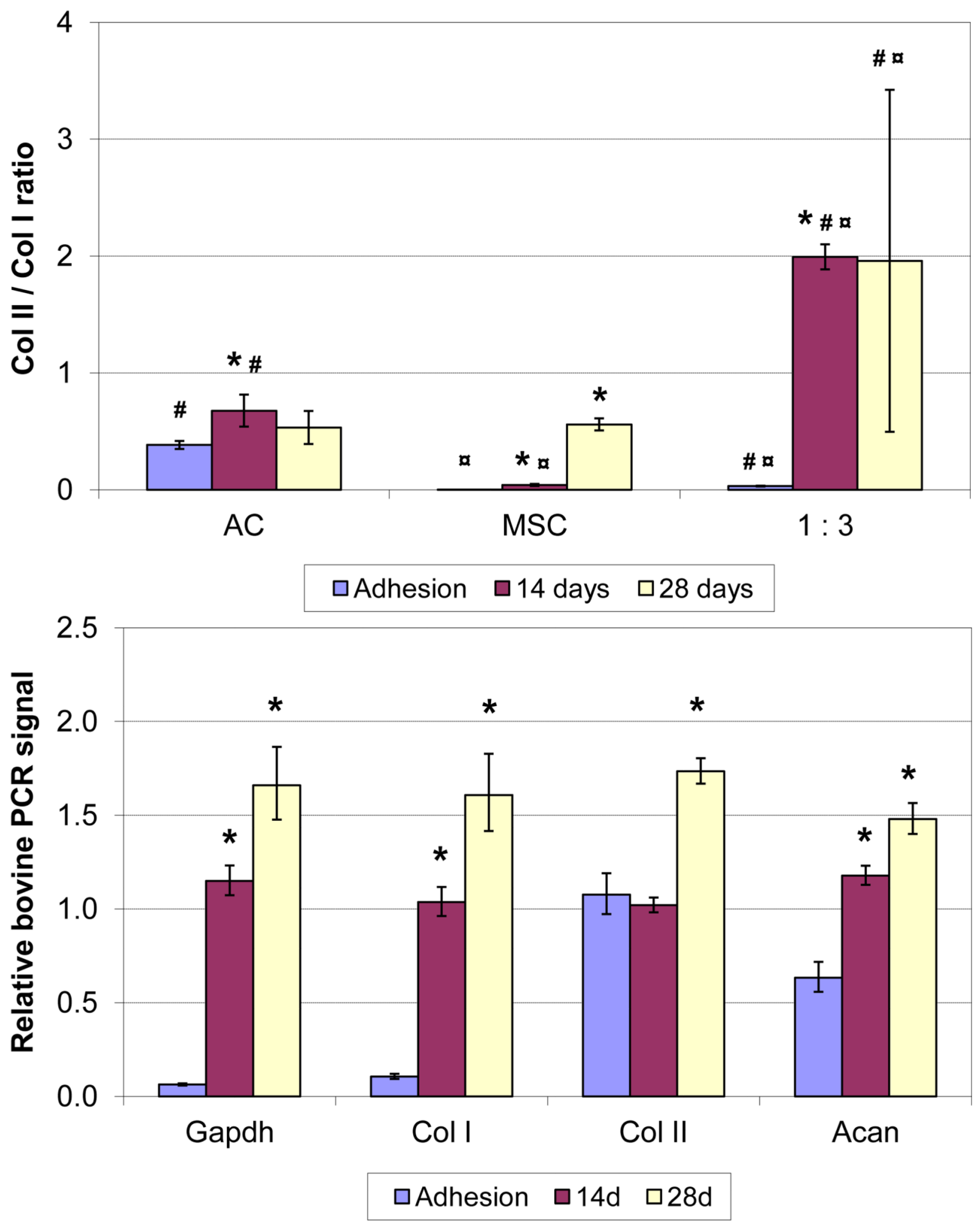

Figure 5.

Ratio of bovine specific-to-non specific quantitative PCR signal. * denote statistically significant difference to the previous time point $(p<0.05)$. 
Meretoja et al.

Page 20

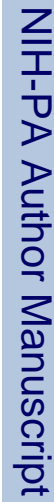

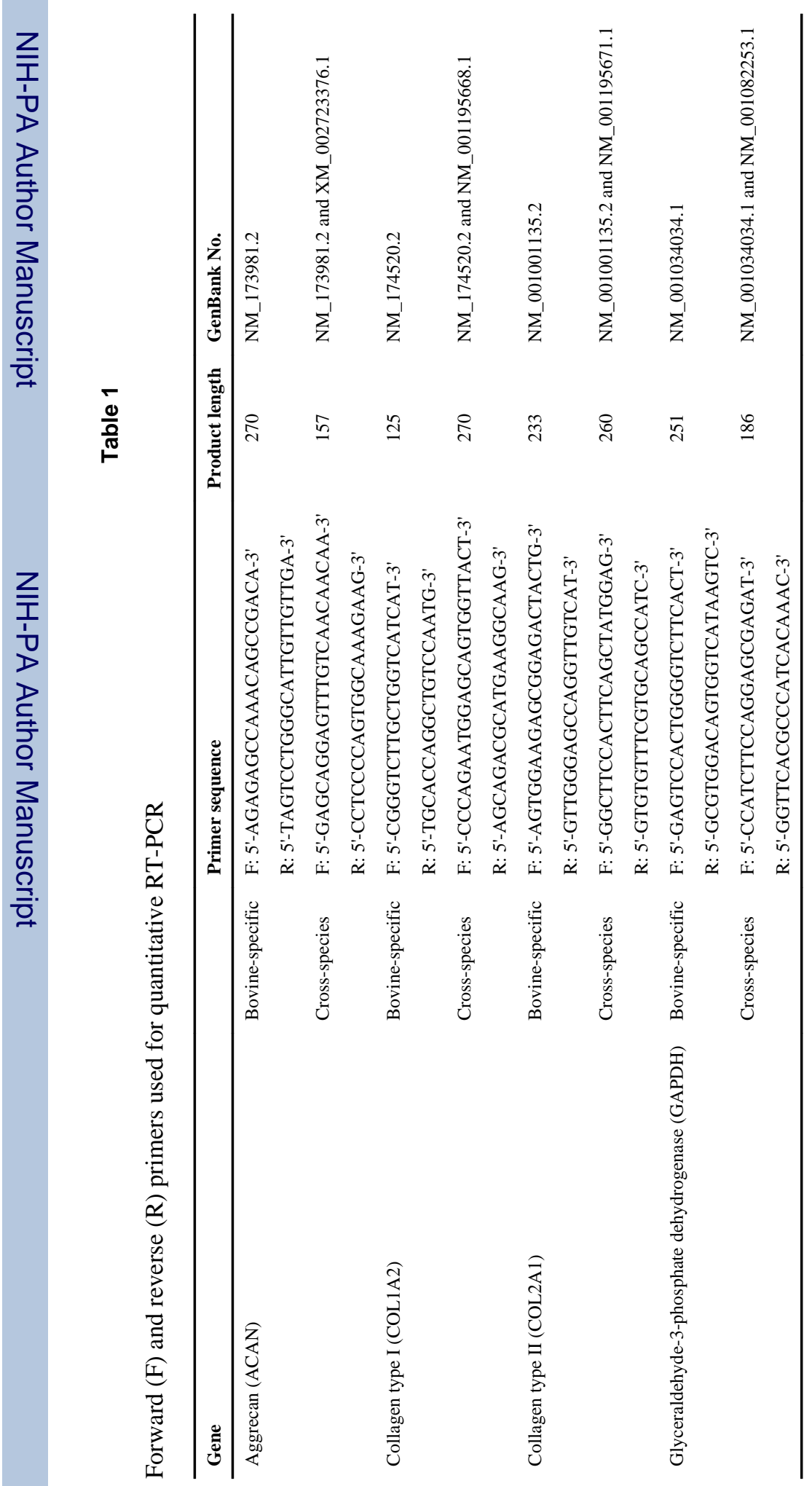

Biomaterials. Author manuscript; available in PMC 2013 September 01. 